\title{
Acoustic Quality and Health in Urban Environments - The SALVE Project
}

\author{
Susanne Moebus, Robynne Sutcliffe, Bryce Lawrence, Salman Ahmed, Timo Haselhoff, Dietwald Gruehn
}

(Prof. Dr. rer. nat. Susanne Moebus, Center for Urban Epidemiology (CUE), Institute for Medical Informatics Biometry and Epidemiology University Colonia Haus Room 3.16 Zweigerstr. 3745130 Essen Germany, susanne.moebus@uk-essen.de) (Dr. rer. medic Robynne Sutcliffe, Center for Urban Epidemiology (CUE), Institute for Medical Informatics Biometry and Epidemiology University Colonia Haus Room 3.16 Zweigerstr. 3745130 Essen Germany, robynne.sutcliffe@uk-essen.de)

(M.Sc. Salman Ahmed, Center for Urban Epidemiology (CUE), Institute for Medical Informatics Biometry and Epidemiology University Colonia Haus Room 3.16 Zweigerstr. 3745130 Essen Germany, salman.ahmed@uk-essen.de)

(Dr.-lng. Bryce Lawrence, Department of Landscape Ecology and Landscape Planning, TU Dortmund University School of Spatial Planning Campus South GB III Room 3.320 August-Schmidt-Str. 1044227 Dortmund Germany, bryce.lawrence@udo.edu)

(B.A. Timo Haselhoff, Center for Urban Epidemiology (CUE), Institute for Medical Informatics Biometry and Epidemiology University Colonia Haus Room 3.16 Zweigerstr. 3745130 Essen Germany, timo.haselhoff@uk-essen.de)

(Prof. Dr. -lng. Dietwald Gruehn, Department of Landscape Ecology and Landscape Planning, TU Dortmund University School of Spatial Planning Campus South GB III Room 3.320 August-Schmidt-Str. 1044227 Dortmund Germany, dietwald.gruehn@udo.edu)

\section{ABSTRACT}

Background sounds of urban regions have been a concern of architecture and construction engineering for years. In the context of health research however, sound has been restricted to the health risk factor noise, thus reduced to sound decibel levels. Accordingly, noise mitigation measures aim exclusively at the reduction of noise level below a certain threshold. Soundscapes on the other hand, comprise all acoustic events of the natural, physical and human environment, which are determined by sound level, frequency, time and space. Soundscape Ecology which includes the study of spatio-temporal heterogeneity of sounds in different landscapes, provides a suitable methodical approach to analyse the relationships between soundscapes, the built environment and human health.

This paper presents SALVE (Acoustic Quality and Health in Urban Environments), a two year interdisciplinary pilot project that started in October 2018 and involves the disciplines of public health and spatial planning. The project aims at the identification of criteria for health-promoting soundscapes in cities. By making year long direct and automated auditory measurements of a robust landuse sample in the city of Bochum, located in the highly urbanized Ruhr Area of Germany, one of the largest multi-seasonal urban soundscape datasets will be generated. These data will be merged with health data from the longitudinal, population-based Heinz Nixdorf Recall (HNR) study. Spatio-statistical models will be further developed to analyse health effects of different types of soundscapes in urban neighborhoods. The project serves as a starting point for an innovative and comprehensive approach to understanding the effects of sound quality on urban public health beyond noise protection. Additionally, knowledge will be gained for the development of solution based health-promoting strategies in spatial planning.

Keywords: HNR, Bochum, urban, health, soundscapes

\section{INTRODUCTION}

\subsection{Noise, Sound and Health}

Rapid urbanisation will reduce or even eliminate the spatial variation between intentional and unintentional sounds (Van Kempen et al. 2014). Accordingly, the characteristics of urban spaces where people can temporarily withdraw from stress factors such as noise may change or become increasingly scarce (Raimbault \& Dubois 2005, Skanberg \& Öhrström 2002). Noise exposure is a worldwide recognized health risk. Especially in cities, a large part of the population is exposed to noise (Farina et al. 2014). Motorised road, rail and air traffic as well as industry and neighbourhoods are considered the most important sources of noise. Chronic noise exposure causes annoyance and stress (WHO 2011). Most research studies focus on traffic noise report effects on health outcomes like hypertension (Fuks et al. 2011), myocardial infarction (Selander et al. 2009), stroke (Sörensen 2011), atherosclerosis (Kälsch et al. 2014), or depression (Orban et al. 2016).

Noise exposure is distinguished between voluntary and involuntary noise exposure. Effects of involuntary noise exposure are associated with feelings of anger or annoyance (noise annoyance). The underlying con $\neg$ cept is based on the assumption that noise only becomes noise through subjective perception. According $\neg$ ly, both measured noise and noise perception are important exposure parameters in noise research. The perceived noise annoyance is determined by both individual and context-related factors, such 
as socio-economic factors, noise sensitivity or perception of the residential environment. For example, in settings of low-noise facades and inner courtyards, visual attractiveness or increased green spaces, living noise backdrops are rated more positively, thereby reducing the effects of noise on annoyance, sleep or blood pressure (Dzhambov \& Dimitrova 2009).

Noise annoyance as a measure of noise exposure may result in different outcomes and conclusions than physically measured or modeled noise. Noise sensitivity was found to be more strongly associated with depression and anxiety than physically measured noise exposure. However, only 10-20\% of the variance of noise pollution is explained by the typical acoustic sound pressure indicators. The explanation of variance is even lower (5\%) in regard to disease effects (Kang et al. 2016). The vast majority of literature dealing with noise and health effects is recommending strategies for noise reduction and noise protection. Per se noise reduction and protection is necessary and contributes to an important health protection agenda by reducing overall unwanted sound levels, however this approach is not exclusively sufficient to create health promoting urban environments. Less noise does not generally mean more pleasant sound, and thus more peace and tranquility, resulting in better overall health and quality of life. For example, noise reduction might result in residents having to deal with previously undetectable aggressive emotional noises. In addition, the same sound pressure can have a very different sound quality if the dominant frequency is in a different range, such as the difference in biophonies of higher frequency versus anthrophonic noises of lower frequency (Farina 2014).

Skanberg \& Öhrström (2002) demand new approaches for traffic noise reduction and Raimbault \& Dubois (2005) identify that sound pressure measurement is not distinctive enough to decide on the quality of sound, but that other factors are relevant such as perception, meaning and mixture of sound. Dependence on Aweighted decibel levels (decibel values corrected for the range of human hearing) alone cannot help to evaluate different sources of noise in an environment over time due to the single number summarisation of the A-weighted value and the lack of data about frequency distribution over time.

Noise mitigation is essential, however, until today it is not completely clear what is further needed in order to achieve good acoustic quality and preserve those environments that are already characterized by a good sound quality. The concept of Soundscape Ecology which encompasses the study of spatio-temporal heterogeneity of sounds in different landscapes (Pijanowski et al. 2011) provides a suitable methodical approach to analyze the relationships between soundscapes, the built environment and human health.

\subsection{Sound and Soundscapes}

The concept of soundscapes is a promising methodological approach to considerably improve the explanatory power in different urban landscapes (Skanberg \& Öhrström 2002) and at the same time understand human perception towards it (Davies et al. 2012). According to Kang \& Schulte-Fortkamp (2016) the sonic and acoustic environment is the sound from all sources that could be heard in that environment. The sonic and acoustic environments can characterize a city just as much as landuse patterns or urban structure types, and include biophonic sounds such as from birds, geophonic sounds of physical environment such as wind and man-made anthrophonic sounds such as speech, motorised traffic, machines or music. In combination, sound pressure, frequency, time and context all define the so-called soundscapes, which can be understood as mixes of biophonies, geophonies and anthrophonies. Different definitions exist in regard to soundscapes depending on the discipline (Pijanowski et al. 2011). Currently, the most common definition is based on the ISO Standard 'an acoustic environment perceived or experienced and/or understood by a person or people in context' (ISO 12913-1). Regardless of the different definitions, all have in common that the concept of soundscapes goes beyond noise as a pollution (Schulte-Fortkamp 2018). Even more, soundscapes focus on the management of the acoustic environment as a resource that can contribute to human quality of life.

So far, limited research has focussed on desirable and therefore pleasant sound environments assuming its beneficial impacts on health and well-being. A recent systematic review of the literature from 2000-2013 in the soundscape field revealed that aspects determining good urban sound quality and the relationship to human health are missing (Van Kamp et al. 2016). In one of the studies that included soundscapes in the investigation, relaxation, communication, dynamics and spatiality were identified as key factors for the evaluation of urban soundscapes (Kang \& Zang 2010). Within cities, its soundscape can be an important 
predictor which points towards the quality of life of its inhabitants. However, most studies do not consider the impact of the acoustic environment on health, either negatively or positively.

Assuming that pleasant and desirable sounds within the urban environment has a beneficial impact on health and well-being of the population, it could be important for city planning and health research to further include the concept of soundscapes (van Kamp et al. 2016). Especially in cities, the positive effects of soundscapes can be of great interest, as environments of good sound quality are needed.

However, so far there have not been sufficient systematic studies to analyse the effects of soundscapes on health. There is a lack of fundamentals, such as evidence-based, health-related soundscape indices, as well as spatio-temporal descriptions of soundscapes (indices) and sufficiently large study cohorts to enable reliable conclusions to be made about their effects.

\section{AIMS AND OBJECTIVES}

This paper presents the background, objectives and methodological approach of SALVE (Acoustic Quality and Health in Urban Areas - Analysis of the interrelationships between soundscapes and health), a two years interdisciplinary pilot project that started at the end of 2018 and involves public health and spatial planning.

Overall, we aim to identify criteria for health-promoting soundscapes, to analyse associations between human health and soundscapes in different urban spatial settings and to develop solutions for a healthpromoting spatial and urban planning.

As a pilot project SALVE intends to provide initial work by developing the basics for reliable methods. Main objectives of the pilot project are to investigate whether soundscapes can be quantified and representative for a gradient of urban landscapes and to determine if those identified soundscapes are associated with different health outcomes, such as depressive symptoms, hypertension and sleep disorders. Following steps are planned: 1. Extensive collection of spatially and temporarily varying soundscapes in the city of Bochum, Germany considering landuse structures (land use types, green spaces, streets, etc.) as well as temporal changes (time of the day and year). 2. Development of an automated widened array of metrics (WAM) for classification of soundscape data into soundscape indicees. 3. Survey to determine soundscape preferences of inhabitants. 4. Analysis of the association between soundscape metrics and health outcomes taking into account socio-demographic data.

\section{STUDY DESIGN}

The sound measurement comprises an extensive typologically organized collection of spatially and temporarily varying soundscapes in the city of Bochum, located in the highly urbanized Ruhr Area, in Western Germany. The raw sound data will be transformed into various soundscape metrics. Comprehensive spatial data from various data sources (e.g. LANUV) shall be integrated. Lastly, health and social data from the already georeferenced longitudinal Heinz Nixdorf Recall (HNR) study will be used. All processed datasets will be transferred to a databank for statistical analyses (Fig. 1).

\subsection{Heinz Nixdorf Recall study}

Participants of the SALVE study will be recruited from the population-based and long-term Heinz Nixdorf Recall (HNR) study, initiated in the year 1999. It is a large epidemiological cohort study conducted in the Cities of Bochum, Mülheim and Essen, located in the densely populated Ruhr area. The study is designed as an individual based observational study with a sample size of 4814 men and women, aged 45-74 years. The participants were drawn from the mandatory registries of the three cities. The study area covers a region of $600 \mathrm{~km} 2$ with 1.2 million inhabitants.

The baseline recruitment started in December 2000 and ended in August 2003. After the baseline measurements, the participants were followed over a period of 15 years, with two consecutive examination periods (after 5 and 10 years) as well as annual mailed questionnaire asking for diseases and medical treatments. In order to be eligible for the SALVE study, the following inclusion criteria were applied: (i) Participant in the HNR study in the third follow up (2011-2015), (ii) Participant residing in the city of Bochum. 


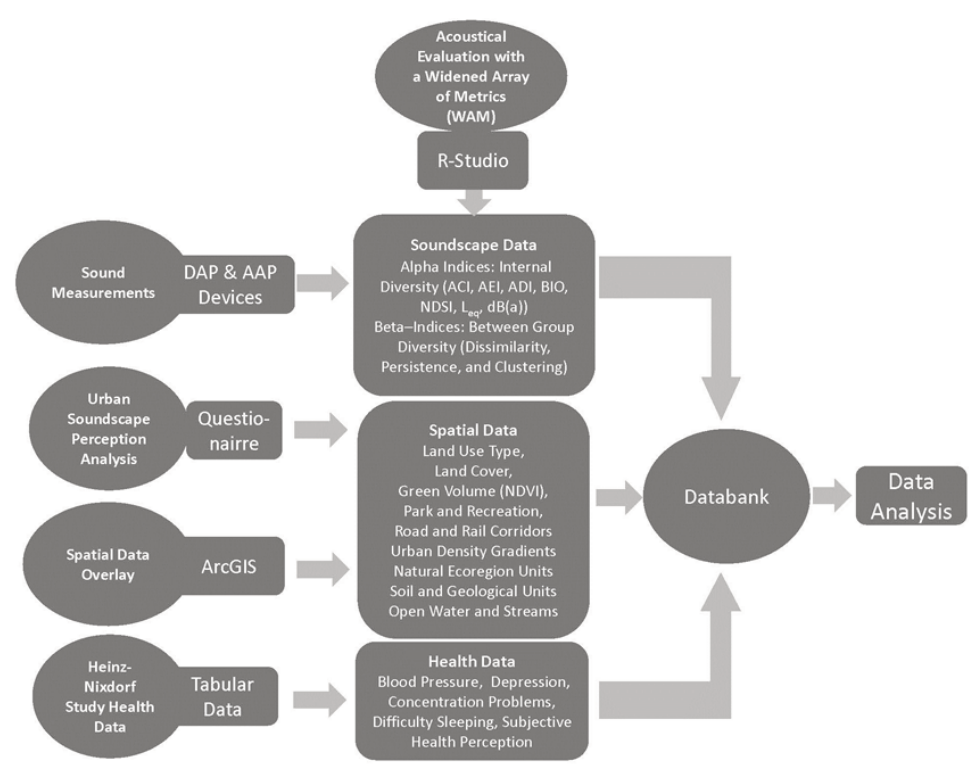

Fig. 1: Multi-level data collection and processing in SALVE

\subsection{Health Data}

For the purpose of the SALVE study, seven health-related variables - mental health, neighborhood satisfaction, blood pressure, sleep medication intake, smoking behavior, Body Mass Index (BMI) and comorbidity - were obtained from the HNR study. Depressive symptomatic was defined as a combination of the intake of anti-depressive medication from the Anatomical Therapeutic Chemical (ATC) groups N06A or N06CA and a depression score using the German 15-item version of the Center for Epidemiologic Studies Depression questionnaire (CES-D). Neighborhood satisfaction was assessed by the question how satisfied are you with your residential area from the Diet and Social Life questionnaire of the HNR study. High blood pressure was defined as the combination of intake of blood pressure medication and presence of hypertension i.e. a blood pressure greater than 140/90, which has been measured by trained personel in the study center. Sleep medication was assessed during the medical interview. BMI was calculated by the measured participant's weight in kilograms divided by the square of height in meters. Comorbidity was defined as the co-occurrence of any combination of the following medical conditions within one participant: cancer, diabetes, asthma, heart attack, heart valve defect, congenital heart defect, stroke, arthrosis, herniated disk, migraine and inflammatory joint disease. Along with the medical assessment, the participants of the HNR study answered questions according to education, marital status, income and working. All participants gave written informed consent prior the study.

\subsection{Soundscape Data}

To record sound, two types of devices are deployed: automatic devices that follow an automated aural procedure (AAP) and manual devices that follow a direct aural procedure (DAP). The state of the art in soundscape research promotes the AAP approach because devices can record sounds over daily, monthly, seasonal, or annual periods, thereby providing dense datasets describing soundscapes over time. However, the AAP approach requires a large number of devices which are expensive and are not suitable for all locations depending on existing infrastructure upon which to mount the devices. The DAP approach can quickly and easily be deployed to the field with minimal intrusion, but are only capable of providing a snapshot of sound in an area and also requires personel for comprehensive field measurements.

This study applies a mixed AAP and DAP approach, placing AAP devices strategically in the field to capture a large number of long term soundscape measures in all landuse categories within the study area, but within a limited number of observations for each landuse category (fig. 2). The DAP approach is used to gather a more temporally-limited number of observations but over a much larger and statistically representative number of landuses in the study area. This approach provides a temporally detailed but geographically limited AAP baseline of longitudinal sound data over one year for all landuse types and then a temporally limited but geographically detailed sound dataset with the DAP approach to understand how different locations within the study area deviate from the baseline of AAP recordings. 


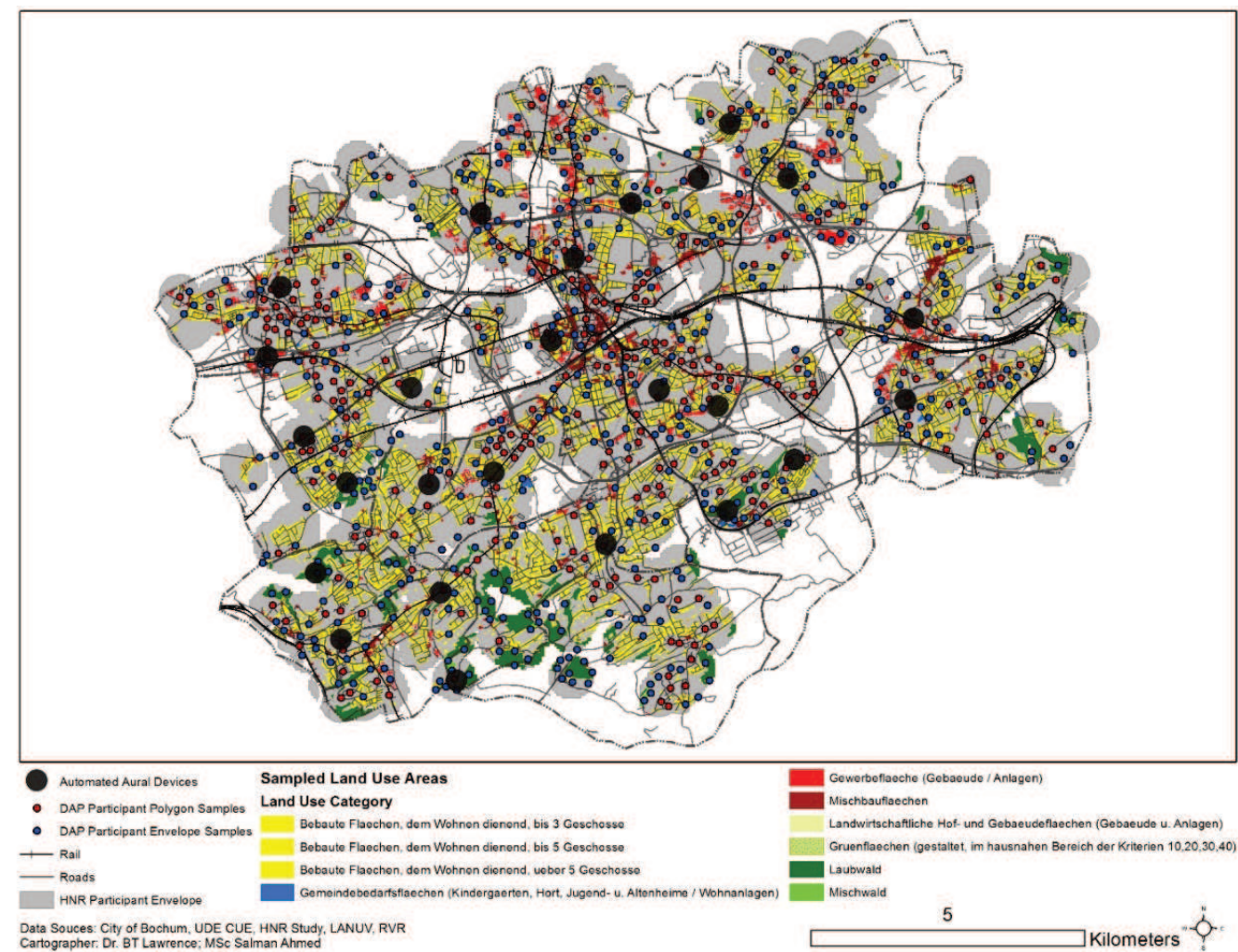

Fig. 2: Sample design summary map of automated aural procedure (AAP) and direct aural procedure (DAP).

A random stratified sample that prioritizes polygons with participants of the Heinz Nixdorf Recall (HNR) study locations was applied to locate all DAP field points. The target population for field recordings was chosen to maximize the spatial proximity of health reporting with field sound recordings so as to capture the soundscape of participant locations. In order to ensure a representative sample from the 10 residential and greenspace landuse types in Bochum, a sample from the total population of Bochum was calculated, which includes more than eleven thousand individual landuse polygons in Bochum. A sample size was calculated for each of the 10 landuse types to the $95 \%$ confidence level, resulting in 730 samples distributed amongst all ten landuse types. The DAP devices will be used to record four times a year (each season) for 5 minutes anytime at different locations on public ground within the city of Bochum. Therefore, more then 130 field days are necessary to achieve the planned number of measurements.

Using 24 AAP recording devices placed stationary in the field, the surrounding sound will be recorded for 5 minutes each hour for 365 days. Of these, 21 AAP recording devices will be placed on private grounds of HNR study participants. The participants will be contacted and informed by telephone as well as by leaflets and brochure. The participants will be asked to sign a consent form. Every two months the data will be downloaded onto a mobile hard drive for physical transfer. The remaining three devices will be placed on public greenspace in Bochum. Location for the AAP devices were selected using an equal number of observations for each landuse type. Given the limitation of 24 total AAP devices, one AAP device was located in each of the three different categories of greenspaces adjacent to built-up area, deciduous forest, and mixed forest. Considering the emerging nature of soundscape research in urban areas, theAAP devices placed in greenspaces will provide an understanding of the differences between soundscapes in built-up areas versus soundscapes in green areas near to or adjacent to built-up areas.

\subsection{Spatial Parameters}

Spatial parameters such as proximity of recording points to different features such as highways, city centers, and airports, availability of surrounding green areas and landuse mix will be incorporated. In addition, other spatial datasets may include built area index (NDBI), park and recreation areas, road and rail corridors, urban density gradients (Corine Urban Atlas), natural ecoregion units, soil and geological units, elevation, physiographic regions and sub-regions, demographic and economic data of the general population, open water and streams, and bus and mass transit stations. 


\subsection{Perception of soundscapes - 'Landscape Theatre'}

The tool 'Landscape Theatre' is used to assess the preference of participants for measured sounds, recruited as a convienent sample via press and radio. Participants rank their perception preferences for different sounds using a Likert scale and the software Turning Point.

\subsection{Data protection}

The study will be conducted in compliance with German Data Protection Act. In accordance with the recommendations of the Federal Office for Information Security (BSI), unauthorized access will be prevented with high levels of information security. Members of the study will be required to sign the data privacy policy and act in accordance with data protection and professional discretion.

\section{CONCLUSION}

As this is a pilot project we will be testing possibilities and approaches during different stages of the implementation phase. In any case, the pilot study will achieve important preliminary results that will allow the description of the quality of soundscapes in a comprehensive manner by means of a diverse set of indicees and the investigation of possible implications of urban soundscapes for a healthy urban environment.

The novelty of this study derives from the idea of treating urban landuse as a so-called "human habitat", and attempting to use sound as an indicator of habitat quality, similar to the use of bioacoustic indices as a measure of the quality of non-human habitats. Furthermore, a temporally longitudinal set of recordings will be systematically gathered from the urban environment with both automated and direct recordings, which will ultimately become one of the largest soundscape dataset ever created in Germany, and can be used for a wide array of follow-up studies. Overall, the application of soundscape metrics in urban environments will provide a deeper understanding of sound than traditional decibel focused metrics used in noise-related studies.

\section{REFERENCES}

Davies, W.J., Adams, M.D., Bruce, N.S., Cain, R., Carlyle, A., Cusack, P., Hall, D.A., Hume, K.I. et al. (2013): Perception of soundscapes: An interdisciplinary approach. Applied Acoustics 74,224-31

Dzhambov AM, Dimitrova DD. Urban green spaces' effectiveness as a psychological buffer for the negative health impact of noise pollution: a systematic review. Noise \& Health. 2014;16(70):157-65.

Farina A, James P, Bobryk C, et al. J. Low cost (audio) recording for advancing soundscape ecology towards the conservation of sonic complexity and biodiversity in natural and urban landscapes. Urban Ecosystems. 2014;17:923-44

Fuks, K., Moebus, S., Hertel, S., Viehmann, A., Nonnemacher, M., Dragano, N., Mohlenkamp, S., et al. (2011): Long-term urban particulate air pollution, traffic noise, and arterial blood pressure. Environmental health perspectives. 119, 1706-11.

Kälsch, H., Hennig, F., Moebus, S., Mohlenkamp, S., Dragano, N., Jakobs, H., Memmesheimer, M., Erbel, R., Jockel, K.H., Hoffmann, B. (2014): Are air pollution and traffic noise independently associated with atherosclerosis: the Heinz Nixdorf Recall Study. European heart journal. 35, 853-60.

Kang J, Aletta F, Gjestland TT, Brown LA, Botteldooren D, Schulte-Fortkamp B, et al. (2016). Ten questions on the soundscapes of the built environment. Building and Environment.108:284-94

Kang J, Schulte-Fortkamp B (eds). Soundscape and the Built Environment. CRC Press Taylor \& Francis Group, 2016

Kang J, Zhang M (2010): Semantic differential analysis of the soundscape in urban open public spaces. Building \& Environ.45,150-7

Orban E, McDonald K, Sutcliffe R, Hoffmann B, Fuks K.B, Dragano N, Viehmann A, Erbel R, Jöckel K-H, Pundt N, Moebus S. (2016): Residential Road Traffic Noise and High Depressive Symptoms after Five Years of Follow-up: Results from the Heinz Nixdorf Recall Study. Environmental health perspectives. 124, 578-85.

Pijanowski, B.C., Villanueva-Rivera, L.J., Dumyahn, S.L., Farina, A. Krause, BL et al. (2011): Soundscape Ecology: The Science of Sound in the Landscape. BioScience. 61, 203-16.

Raimbault, M., Dubois, D. (2005): Urban soundscapes: Experiences and knowledge. Cities 22, 339-50

Raimbault, M., Dubois, D. (2005): Urban soundscapes: Experiences and knowledge. Cities. 22, 339-50.

Schulte-Fortkamp B (ed). Soundscapes, Standardization, and Application. Euronoise 2018 - Conference proceedings; 2018; Crete

Selander, J., Nilsson, M.E., Bluhm, G., Rosenlund, M., Lindqvist, M., Nise, G., Pershagen, G. (2009): Long-term exposure to road traffic noise and myocardial infarction. Epidemiology. 20, 272-9

Skanberg, A., Öhrström, E. (2002): Adverse health effects in relation to urban residential soundscapes. J Sound Vibration 250, 151-5

Sorensen, M., Hvidberg, M., Andersen, Z.J., Nordsborg, R.B., Lillelund, K.G., Jakobsen, J., Tjonneland, A., Overvad, K., Raaschou-Nielsen, O. (2011): Road traffic noise and stroke: a prospective cohort study. European heart jnl. 32, 737-44.

Van Kamp, I, Klaeboe, R., Kruize, H., Brown, A.L., Lercher, P. (eds). Soundscapes, human restoration and quality of life. INTERNOISE and NOISE-CON Congress and Conference Proceedings; 2016: Institute of Noise Control Engineering.

Van Kempen E, Devilee J, Swart W, van Kamp I. Characterizing urban areas with good sound quality: Development of a research protocol. Noise and Health. 2014;16:380-7

WHO Regional Office for Europe. (2011): Burden of disease from environmental noise. Quantification of healthy life years lost in Europe. Available from: www.euro.who.int/_data/assets/pdf_file/0008/136466/e94888.pdf. Last access 31.12.2018 